Aim of the study: The aim of this retrospective study was to determine the prognostic impact of epidermal growth factor receptor (EGFR) expression changes during neoadjuvant chemoradiotherapy in patients with locally advanced rectal cancer.

Material and methods: Fifty patients with locally advanced rectal cancer were evaluated. All the patients were administered the total dose of $44 \mathrm{~Gy}$. Capecitabine has been concomitantly administered in the dose $825 \mathrm{mg} / \mathrm{m}^{2}$ in two daily oral administrations. Surgery was indicated 4-8 weeks from the chemoradiotherapy completion. Epidermal growth factor receptor expression in the pretreatment biopsies and in the resected specimens was assessed with immunohistochemistry. Results: All of 50 patients received radiotherapy without interruption up to the total planned dose. In $30 \mathrm{pa}$ tients sphincter-saving surgery was performed, 20 patients underwent amputation of the rectum. Downstag ing was described in 30 patients. Four patients have had complete pathologic remission. Twenty-six patients have had partial remission, the disease was stable in 15 patients. Progression was reported in 5 patients. The median disease-free survival was 64.9 months, median overall survival was 76.4 months. Increased EGFR expression was found in 12 patients (26.1\%). A statistically significantly shorter overall survival $(p<0.0001)$ and disease-free survival $(p<0.0001)$ was found in patients with increased expression of EGFR compared with patients where no increase in the expression of EGFR during neoadjuvant chemoradiotherapy was observed. Conclusions: The overexpression of EGFR during neoadjuvant chemoradiotherapy for locally advanced rectal adenokarcinoma associated with significant shorter overall survival and disease free survival.

Key words: rectal adenocarcinoma, neoadjuvant treatment, radiotherapy chemotherapy, epidermal growth factor receptor.

Contemp Oncol (Pozn) 2015; 19 (1): 48-53 DOI: $10.5114 /$ wo. 2015.50013

\section{The prognostic significance of tumor epidermal growth factor receptor (EGFR) expression change after neoadjuvant chemoradiation in patients with rectal adenocarcinoma}

Igor Richter ${ }^{1}$, Josef Dvořák ${ }^{2}$, Marek Urbanec ${ }^{3}$, Antonin Bluml ${ }^{4}$, Eva Čermáková5, Jiří Bartoš́, Jiří Petera

\author{
1Department of Oncology, Regional Hospital Liberec, Liberec, Czech Republic \\ 2Department of Oncology and Radiotherapy, Charles University, Medical School \\ and Teaching Hospital, Hradec Kralove, Czech Republic \\ ${ }^{3}$ Department of Pathology, Ceska Lipa Hospital, Ceska Lipa, Czech Republic \\ ${ }^{4}$ Department of Medical Biophysics, Charles University, Medical School and Teaching \\ Hospital, Hradec Kralove, Czech Republic \\ Department of Medical Biophysics, Charles University, Medical School and Teaching \\ Hospital, Hradec Kralove, Czech Republic \\ ${ }^{6}$ Department of Oncology, Regional Hospital Liberec, Liberec, Czech Republic
}

\section{Introduction}

Malignant tumours of the colon and rectum are the most common cancers in developed countries. The incidence of rectal adenocarcinoma represents approximately $30 \%$ of this number. A neoadjuvant chemoradiotherapy followed by total mesorectal excision is the basic procedure of the treatment for patients with locally advanced rectal cancer. Neoadjuvant chemoradiotherapy has shown a lower incidence of local recurrence and better toxicity profile compared to adjuvant therapy, but no survival benefit was shown [1]. Potentiation with 5-fluorouracil or capecitabine has shown a higher percentage of pathological complete remission and a lower percentage of local recurrence compared to the treatment with radiotherapy alone [2-5]. The development of molecular biology enables us to look for other predictive factors of overall outcomes. One of them is receptor for epidermal growth factor (EGFR). The overexpression of EGFR is observed in $50-80 \%$ of rectal carcinomas and is associated with a worse prognosis [6-8]. Radiobiological EGFR studies confirm the critical role of cytoprotective and pro-proliferative responses of tumour cells after irradiation. The increase in EGFR expression after radiotherapy is related to accelerated repopulation of cancer cells $[9,10]$. Increased tumour repopulation during radiotherapy leads to the recovery of clonogenic tumour cells, thereby causing counter productivity to radiation therapy alone [11-13]. Based on the above information, the inhibition of EGFR function during cancer treatment is one of the most investigated processes. Monoclonal antibodies against EGFR have reached the greatest expansion. Cetuximab and panitumumab are used in the treatment of metastatic colorectal cancer [14-18]. Neoadjuvant treatment of rectal adenocarcinoma has been the topic of several clinical papers evaluating the benefits of monoclonal antibodies against EGFR combined with chemoradiotherapy. Most dates are for cetuximab [19-21]. Conversely, the percentage of pathological complete response ( $p C R$ ) was low (5-8\%) compared to the percentage of pCR in separate chemoradiotherapy. According to a meta-analysis of phase II and III in clinical trials 
in 3157 patients, pCR was described in $13.5 \%$ of treated patients with neoadjuvant chemoradiotherapy [22]. Panitumumab was evaluated in a phase II clinical study in the neoadjuvant treatment of rectal adenocarcinoma. A total of 60 patients were evaluated. The percentage of complete pathologic remissions was $21.1 \%$ [23]. Therefore more options of how to better individualise the treatment of patients with EGFR inhibitors are being looked for. The aim of this retrospective study was to determine the prognostic impact of EGFR expression changes during neoadjuvant chemoradiotherapy in patients with locally advanced rectal cancer, by comparison of EGFR expression in pretreatment endoscopic biopsies and resection specimens after neoadjuvant chemoradiotherapy.

\section{Material and methods}

Between January 2005 and December 2009 a total of 59 patients were treated with preoperative radiation for rectal adenocarcinoma potentiated with capecitabine in the Department of Oncology, Liberec Hospital. Fifty patients, 34 men and 16 women, were evaluated. Nine patients were not evaluated because of incomplete clinical and pathological data. The mean age was 61.4 years (range 40-78 years). Microscopically, tubular adenocarcinoma was identified in all 50 patients. Mucinous component was described in three patients. Histologically, the tumour was a well-differentiated adenocarcinoma in 3 patients, moderately differentiated in 38 patients, and poorly differentiated in 9 patients. As far as the anatomical site is concerned, 24 patients had a distal tumour margin localised as far as $5 \mathrm{~cm}$ from the internal sphincter, and the same number of patients had between 5.1 and $10 \mathrm{~cm}$. The case of the distal edge of the tumour penetrating more than $10 \mathrm{~cm}$ was described in two patients. Before the neoadjuvant chemoradiotherapy was started, 28 patients were in the second clinical stage and 22 patients in the third clinical stage, according to TNM classification.

\section{Treatment}

The source of radiation was a linear accelerator Elekta Precise or Elekta Synergy (Elekta, Sweden). We used ionising photon radiation with an energy of $15 \mathrm{MeV}$. Patients were irradiated using the $3 \mathrm{D}$ conformal radiotherapy technique, or IMRT, using segmented fields. All the patients were administered a total dose of 44 Gy (fractionation of 2 Gy) in 22 fractions to the tumour area, mesorectum, and pelvic regional lymph nodes [24]. Capecitabine was concomitantly administered with a dosage of $825 \mathrm{mg} / \mathrm{m}^{2}$ in two daily oral administrations for the whole duration of radiotherapy, including weekends. Surgery was indicated at intervals of 4-8 weeks from the completion of chemoradiotherapy.

\section{Immunohistochemical determination of epidermal growth factor receptor}

The evaluation was semi-quantitative, and the colour intensity of at least $1 \%$ of tumour cells was assessed as follows: $0=$ none, $1+=$ mild, $2+=$ moderate, $3+=$ severe (Figs. 1-3). A commercial kit (EGFR PharmDxTM, Dako, Denmark) was used. Slides were evaluated by an expe-

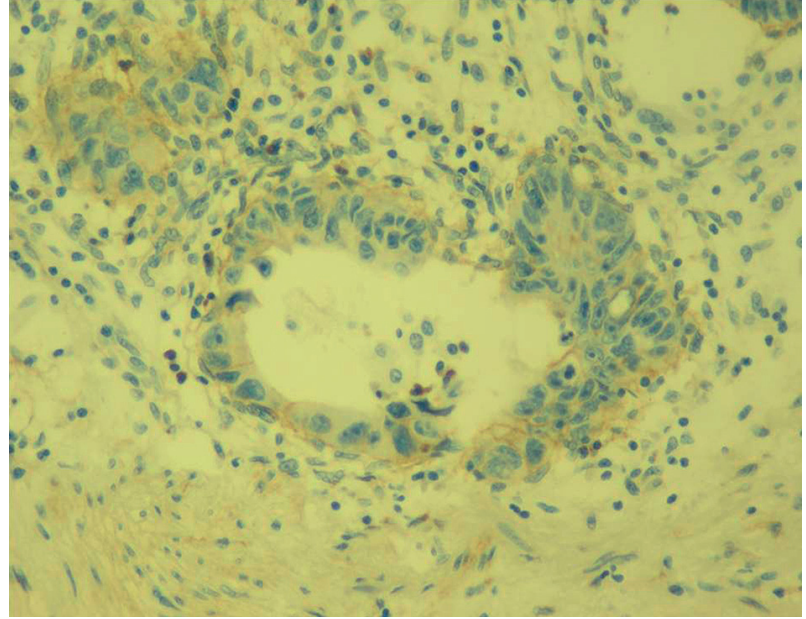

Fig. 1. EGFR expression 1+

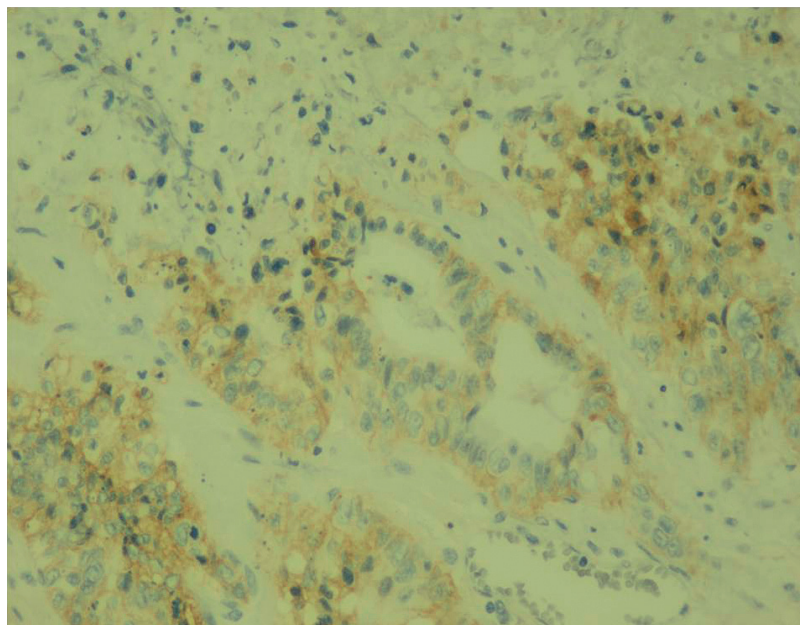

Fig. 2. EGFR expression 2+

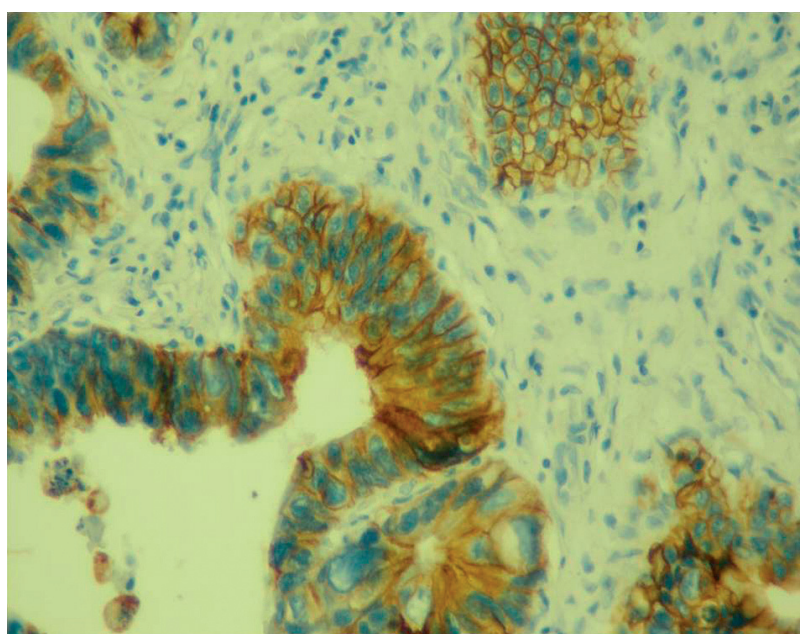

Fig. 3. EGFR expression 3+

rienced pathologist who was not familiar with the treatment results of the patients. Endobioptic findings before treatment as well as resection specimens after neoadjuvant chemoradiotherapy and surgical treatment were analysed in our patient group. 
Table 1. Epidermal growth factor receptor (EGFR) expression score in biopsies and resected specimens

\begin{tabular}{|ccc|}
\hline $\begin{array}{c}\text { EGFR } \\
\text { expression score }\end{array}$ & $\begin{array}{c}\text { EGFR expression } \\
\text { in pretreatment } \\
\text { biopsies } \boldsymbol{N}(\%)\end{array}$ & $\begin{array}{c}\text { EGFR expression } \\
\text { in resected } \\
\text { specimen } \boldsymbol{N}(\%)\end{array}$ \\
\hline 0 & $22(44)$ & $23(46)$ \\
\hline $1+$ & $18(36)$ & $8(16)$ \\
\hline $2+$ & $5(10)$ & $11(22)$ \\
\hline $3+$ & $5(10)$ & $4(8)$ \\
Not applicable & $0(0)$ & $4(8)$ \\
\hline
\end{tabular}

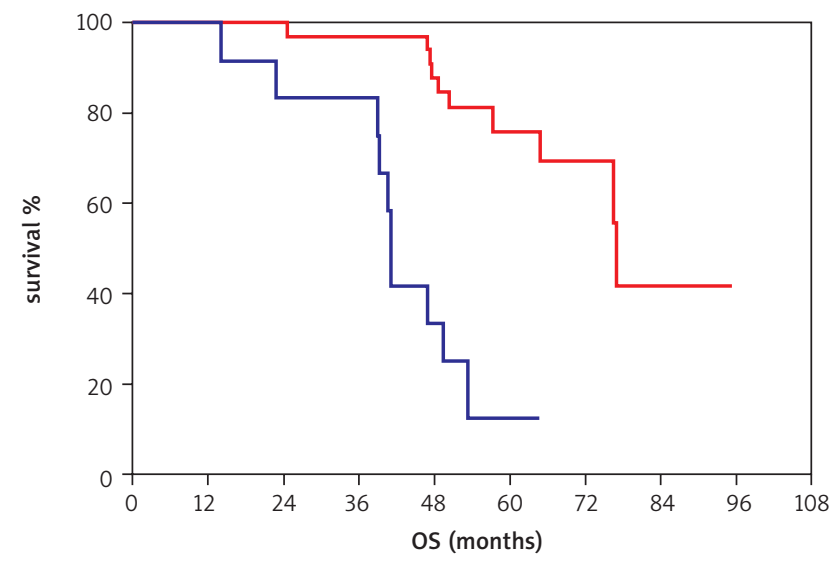

Fig. 4. Overall survival in months (red curve: patients without increase of EGFR expression, blue curve: patients with increase of EGFR expression)

\section{Statistical analysis}

The statistical evaluation was performed using the Number Cruncher Statistical Systems 9 NCSS (Kaysville, Utah, USA) program. Overall survival (OS) = time from the first histological verification until death or the date of the last check for survivors. Disease-free survival (DFS) = time from surgery to distant or local recurrence or the last control of a patient without recurrence. The overall survival and disease-free survival was assessed using Kaplan-Meier analysis. The impact of EGFR expression on treatment outcomes (OS, DFS) was assessed by the log-rank test. All the statistical tests were performed at the significance level $\alpha=0.05$.

\section{Results}

All of the 50 patients received radiotherapy without interruption up to the total planned dose. No patient died during the treatment. Concomitant chemotherapy was discontinued prematurely in four patients because of haematological and gastrointestinal toxicity. No patient was hospitalised because of acute treatment toxicity. Non-haematological toxicity evaluation did not achieve grade III or IV. Anaemia grade III was found in one patient. The median time between chemoradiotherapy completion and surgery was 44 days (6.3 weeks). In 30 patients sphincter-saving surgery was performed, and 20 patients underwent amputation of the rectum. RO resection was performed in 47 patients, and microscopically positive margin was de- scribed by a pathologist in 3 patients. There was no surgically macroscopic residue left in any patient. According to the pathological TNM classification, 14 patients were at the first clinical stage, 24 patients in the second clinical stage, and 8 patients in the third clinical stage after the operation. Four patients achieved complete pathological remission. Complete pathological response was defined as the absence of tumour tissue in the specimen. No patient had the generalisation of the disease described intraoperatively. Downstaging was described in 30 patients. Four patients had complete pathologic remission. Twenty-six patients had partial remission. The disease was stable in 15 patients. Progression was reported in 5 patients. At the time of assessment (31 December 2013) median follow-up was 51.3 months. A recurrence occurred in 25 patients, and 25 patients had no signs of recurrence. Local recurrence was found in 8 patients, and generalisation of disease was reported in 17 patients. The most common sites of metastases were the liver (eight patients) and lungs (seven patients). One patient suffered from brain metastases, and metastatic involvement of retroperitoneal lymph nodes was found in one patient. The median DFS was 64.9 months ( $95 \% \mathrm{Cl}: 26.1$ to 67.8 months). The 3-year DFS was $56 \%$. A total of 21 patients died, and 29 patients remained alive. The median OS was 76.4 months $(95 \% \mathrm{Cl}$ : 57.3 to 76.9 months). The 3 -year OS was $92 \%$. Epidermal growth factor receptor expression was examined both by endobiopsy and in resection specimens after neoadjuvant chemoradiotherapy (Table 1). Forty-six patients were enrolled into the evaluation of EGFR expression changes. In four patients no change in expression of EGFR was evaluated because pathologic complete response was achieved after neoadjuvant chemoradiotherapy. Increased EGFR expression was found in 12 patients. In 34 patients no increased expression of EGFR was observed (23 patients without any change of EGFR expression, 11 patients with a decrease of EGFR expression). Statistically significantly shorter OS and DFS was found in patients with increased expression of EGFR compared with patients in whom no increase in expression of EGFR during neoadjuvant chemoradiotherapy was observed. The median OS in patients with increased EGFR expression was 41.1 months (95\% $\mathrm{Cl}$ : 39.1 to 47.0 months). The median OS for patients without increased expression of EGFR was 76.9 months (95\% $\mathrm{Cl}: 76.4$ to 76.9 months, log-rank test: $p<0.0001)$. The median DFS in patients with increased EGFR expression was 13.7 months ( $95 \% \mathrm{Cl}: 3.8$ to 15.8 months). The median DFS in patients without increased EGFR expression was 67.8 months (95\% Cl: 55.7 to 67.8 months, log-rank $p<0.0001)$. Kaplan-Meier curves are presented in Figures 4 and 5 .

\section{Discussion}

Our retrospective study confirmed its target and proved that patients with increased expression of EGFR during neoadjuvant chemoradiotherapy demonstrate significantly shorter OS and DFS. We demonstrated increased expression of EGFR in 12 patients, i.e. $26.1 \%$ of all evaluated patients. In 2012, a retrospective study examining the effects 
of changes in expression of EGFR in 53 patients treated with neoadjuvant chemoradiotherapy for locally advanced rectal cancer was presented [25]. Radiotherapy was potentiated by the administration of 5 -fluorouracil. The increase of EGFR expression during neoadjuvant chemoradiotherapy was similarly associated with statistically significantly shorter DFS and OS. Both studies prove the prognostic impact of EGFR expression change in two different groups of patients treated in two different cancer centres. Epidermal growth factor receptor was evaluated in different pathology laboratories. In the group of 53 patients, radiotherapy was potentiated by continuous 5-FU, and in our group by capecitabine. In both studies the prognostic significance of EGFR dynamics was confirmed, therefore they cannot be considered to be pure coincidence but a proven link. The prognostic significance of EGFR expression was the topic of other studies, as well. Azria evaluated the effect of EGFR expression on loco-regional recurrence in biopsy on therapeutic results in 77 patients treated with neoadjuvant radiotherapy. Epidermal growth factor receptor positivity was found in $56 \%$ of patients. The median of follow-up of 36 months proved the significantly higher number of local recurrences in patients overexpressing EGFR above $25 \%$ in multivariate analysis (HR 7.18, $p=0.037$ ) [26]. Another clinical paper evaluated 92 patients treated with neoadjuvant chemoradiotherapy for locally advanced rectal cancer. Epidermal growth factor receptor positivity was found in $71 \%$ of patients. Patients with high EGFR expression showed significantly shorter OS ( $p=0.013)$, DFS ( $p=0.002)$, and distant metastases-free survival $(p=0.003)$ compared to patients with no or low expression of EGFR [27]. Conversely, another retrospective study did not show any prognostic impact of EGFR and KRAS mutations expression in 146 patients treated with neoadjuvant chemoradiotherapy for rectal adenocarcinoma on OS and DFS. In this study the determination of the EGFR gene amplification by FISH was done [28]. Reparations, redistribution, repopulation, and reoxygenation belong to the basic mechanisms governing the interaction between ionising radiation and tissues (4R) [29]. Epidermal growth factor receptor plays an important role in all four mechanisms. Epidermal growth factor receptor has an important function in the repair of cellular damage induced by radiation. Epidermal growth factor receptor may also be directly translocated into the cell nucleus with direct activation of transcription factors with cell reparation as a final result [30-32]. Similarly, after EGFR activation by ionising radiation, the signalling pathway of the Ras/Raf/MAPK is activated with the expression of repair genes (Rad51, ATM, XRCC1) [33-35]. Epidermal growth factor receptor also has an influence on the redistribution of cells after irradiation. Epidermal growth factor receptor inhibitors lead to a redistribution of the cell cycle by a block in the G1 phase. Moreover, the cell cycle can also be prolonged by a radiation-induced block in the $\mathrm{G} 2$ phase of the cell cycle [36]. As already mentioned, pathologic complete remission with a combination of EGFR inhibitor and neoadjuvant chemoradiotherapy of locally advanced rectal adenocarcinoma was low. The explanation of this will need further understanding of the interaction between radiotherapy, EGFR inhibitors, and cytostatics.

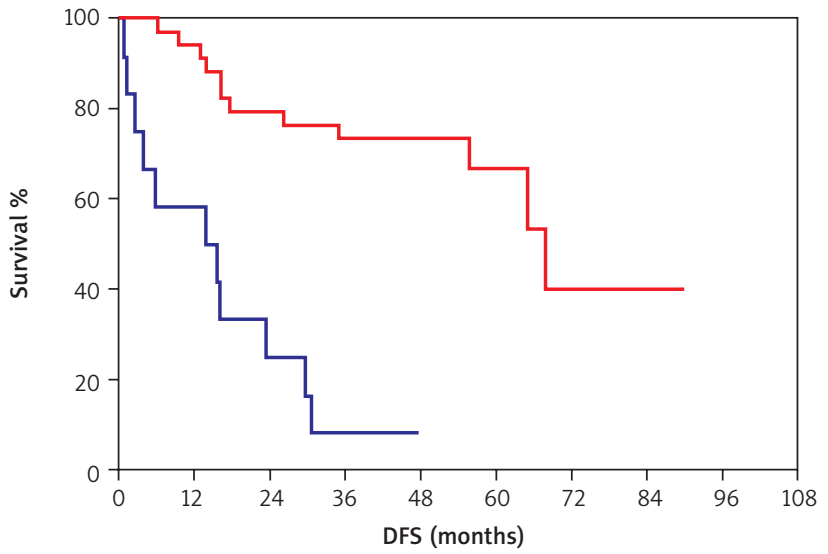

Fig. 5. Disease-free survival in months (red curve: patients without increase of EGFR expression, blue curve: patients with increase of EGFR expression)

Nyati et al. discussed in their paper whether the cause could be seen in the suboptimal sequence of administered treatment that might lead to an antagonistic rather than a potentiating effect [37]. It was found that EGFR inhibitors cause the redistribution of the cell cycle by $\mathrm{G} 1$ phase blockade [36]. Administration of EGFR inhibitors before the cytostatic scan arrested the cell cycle in the G1 phase, which can affect the attenuation of the effects of subsequently administered cytostatics, with an impact on other phases of the cell cycle. It is the cytostatics used in the treatment of colorectal cancer (5-fluorouracil, oxaliplatin, irinotecan) that have the most highlighted effect on the cell cycle in the S/G2/M phase of the cell cycle [19]. The administration of EGFR inhibitors leads to a reduction in tumour proliferation. Conversely, radiotherapy has a lesser effect on the less proliferating tumour cells. This is another cause of lower pathological complete remission after the concomitant treatment with EGFR inhibitors and radiotherapy [38]. The variation in the prognostic significance of EGFR in clinical studies may also be associated with the method of determining the EGFR expression [26, 27, 39, 40]. It can be obtained from a combination of various influences such as the sampling method, preparation of tissue samples, the method of receptor activity evaluation, and others. On the basis of the results of the current retrospective study it would be appropriate to identify a group of patients with increased EGFR expression during neoadjuvant chemoradiotherapy. This group of patients would gain benefit from additional therapy by EGFR inhibitors after surgery. In the future, prospective studies could take advantage not only of immunohistochemistry ex vivo, but also of immunohistochemistry in vivo using PET EGFR, which could evaluate the dynamics of EGFR expression not only before and after chemoradiotherapy, but also during chemoradiotherapy with early antiEGFR therapy [41].

In conclusion, the increase of EGFR expression during neoadjuvant chemoradiotherapy for locally advanced rectal cancer is associated with significantly shorter overall survival and disease-free survival.

The authors declare no conflict of interest. 


\section{References}

1. Sauer R, Liersch T, Merkel S, et al. Preoperative versus postoperative chemoradiotherapy for rectal cancer: results of the German CAO/ARO/AIO-94 randomised phase III trial after a median follow-up of 11 years. J Clin Oncol 2012; 30: 1923-33.

2. Bosset JF, Calais G, Mineur L, et al. Enhanced tumorocidal effect of chemotherapy with preoperative radiotherapy for rectal cancer: preliminary results EORTC 22921. J Clin Oncol 2005; 23: 5620-7.

3. Gérard JP, Conroy T, Bonnetain F, et al. Preoperative radiotherapy with or without concurrent fluorouracil and leucovorine in T3-4 rectal cancers: results of FFCD 9203. J Clin Oncol 2006; 24: 4620-5.

4. Hofheinz RD, Wenz F, Post S, et al. Chemoradiotherapy with capecitabine versus fluorouracil for locally advanced rectal cancer: a randomised, multicentre, non-inferiority, phase 3 trial. Lancet Oncol 2012; 13: 579-88.

5. De Caluwé L, Van Nieuwenhove Y, Ceelen WP. Preoperative chemoradiation versus radiation alone for stage II and III resectable rectal cancer. Cochrane Database Syst Rev 2013; 2: CD006041.

6. Steele RJ, Kelly P, Ellul B, Eremin O. Epidermal growth factor receptor expression in colorectal cancer. Br J Surg 1990; 77: 1352-4.

7. Mayer A, Takimoto M, Fritz E, Schellander G, Kofler K, Ludwig H. The prognostic significance of proliferating cell nuclear antigen, epidermal growth factor receptor, and MDR gene expression in colorectal cancer. Cancer 1993; 71: 2454-60.

8. Khorana AA, Ryan CK, Cox C, Eberly S, Sahasrabudhe DM. Vascular endothelial growth factor, CD68, and epidermal growth factor receptor expression and survival in patients with stage $I$ and stage III colon carcinoma: a role for the host response in prognosis. Cancer 2003; 97: 960-8.

9. Withers HR, Taylor JM, Maciejewski B. The hazard of accelerated tumor clonogen repopulation during radiotherapy. Acta Oncol 1988; 27: 131-46.

10. Baumann M, Petersen C, Eichler W, et al. Mechanism of repopulation in experimental squamos cell carcinoma. In: Kogelnik HD, Lukas P, Sedlmayer F. Progress in radiation-oncology. Vol. 7. Bologna, Monduzzi 2002; 417-22.

11. Begg AC. Prediction of repopulation rates and radiosensitivity in human tumours. Int J Radiat Biol 1994; 65: 103-8.

12. Fowler JF. Rapid repopulation in radiotherapy: a debate on mechanism. The phantom of tumor treatment-continually rapid proliferation inmasked. Radiother Oncol 1991; 22: 156-8.

13. Schmidt-Ullrich RK, Contessa JN, Dent P, Mikkelsen RB, Valerie K, Reardon DB, Bowers G, Lin PS. Molecular mechanism of radiation-induced accelerated repopulation. Radiat Oncol Investig 1999; 7: 321-30.

14. Cunningham D, Humblet Y, Siena S, et al. Cetuximab monotherapy and cetuximab plus irinotekan in irinotekan-refractory metastatic colorectal cancer. N Engl J Med 2004; 351: 337-45.

15. Van Cutsem E, Köhne CH, Láng I, http://www.ncbi.nlm.nih. gov/pubmed/?term=Folprecht\%20G\%5BAuthor\%5D\&cauthor=true\&cauthor_uid=21502544et al. Cetuximab plus irinotecan, fluorouracil, and leucovorine as fist-line treatment for metastatic colorectal cancer: update analysis of overall survival according to tumor KRAS and BRAF station status. J Clin Oncol 2011; 29: 2011-9.

16. Van Cutsem E, Peeters M, Siena S, et al. Open label phase III trial of panitumumab plus best supportive care compared with best supportive care alone in patiens with chemotherapy-refractory metastatic colorectal cancer. J Clin Oncol 2007; 25: 1658-64.

17. Oliner KS, Douillard JY, Siena S, et al. Analysis of KRAS/NRAS and BRAF station in the phase III PRIME study of panitumumab (pmab) plus FOLFOX versus FOLFOX as first-line treatment (tx) for metastatic colorectal cancer (mCRC). J Clin Oncol 2013; 31 (Suppl): Abstract 3511.

18. Krawczyk P, Kowalski DM. Genetic and immune factors underlying the efficacy of cetuximab and panitumumab in the treatment of patients with metastatic colorectal cancer. Contemp Oncol 2013; 17: 413-22

19. Rödel C, Arnold D, Hipp M, et al. Phase I-II trial of cetuximab, capecitabine, oxaliplatin, and radiotherapy as preoperative treatment in rectal cancer. Int J Radiat Oncol Biol Phys 2008; 70: 1081-6.

20. Bertolini F, Chiara S, Bengala C, et al. Neoadjuvant treatment with single-agent cetuximab followed by 5-FU, cetuximab, and pelvic radiotherapy: a phase II study in locally advanced rectal cancer. Int J Radiat Oncol Biol Phys 2009; 73: 466-72.

21. Machiels JP, Sempoux C, Scalliet P, et al. Phase I/II study of preoperative cetuximab, capecitabine, and external beam radiotherapy in patients with rectal cancer. Ann Oncol 2007; 18: 738-44.

22. Hartley A, Ho KF, McConkey C, Geh Jl. Pathological komplete response following preoperative chemoradiotheraoy in rectal cancer: analysis of phase II/III trials. Br J Radiol 2005; 78: 934-8.

23. Pinto C, Di Fabio F, Maiello E, et al. Phase II study of panitumumab, oxaliplatin, 5-fluorouracil, and concurrent radiotherapy as preoperative treatment in high-risk locally advanced rectal cancer patients (StarPan/STAR-02 Study). Ann Oncol 2011; 22: 2424-30.

24. Šlampa P, Lovas P. Lovasová Z, et al. Karcinomy konečníku. In: Šlampa P, et al. Radiační onkologie v praxi. Masarykův onkologická ústav. Brno 2011; 70-8.

25. Dvorak J, Sitorova V, Ryska A, et al. The prognostic significance of changes of tumor epidermal growth factor receptor expression after neoadjuvant chemoradiation in patiens with rectal adenocarcinoma. Strahlenter Onkol 2012; 10: 145-7.

26. Azria D, Bibeau F, Barbier N, et al. Prognostic impact of epidermal growth factor receptor (EGFR) expression on loco-regional recurrence after preoperative radiotherapy in rectal cancer. BMC Cancer 2005; 5 : 62.

27. Li S, Kim JS, Kim JM, Cho MJ, Yoon WH, Song KS, Yeo SG, Kim JS. Epidermal growth factor receptor as a prognostic factor in locally advanced rectal-cancer patients treated with preoperative chemoradiation. Int J Radiat Oncol Biol Phys 2006; 65: 705-12.

28. Bengala C, Bettelli S, Bertolini F, et al. Prognostic role of EGFR gene copy number and KRAS station in patiens with locally advanced rectal cancer treated with preoperative chemoradiotherapy. $\mathrm{Br}$ J Cancer 2010; 103: 1019-24.

29. Withers HR. The 4 R's of radiotherapy. In: Lett JT, Alder H. Advanc es in Radiation Biology. Vol. 5. Academic Press, New York 1975; 241-71.

30. Lin SY, Makino K, Xia W, Matin A, Wen Y, Kwong KY, Bourguignon L, Hung MC. Nuclear localization of EGF receptor and its potential new role as a transcription factor. Nat Cell Biol 2001; 3: 802-8.

31. Oksvold M, Huitfeldt H, Stang E, Madshus I. Localizing the EGF receptor. Nat Cell Biol 2002; 4: E22-3.

32. Waugh MG, Hsuan JJ. EGF receptors as transcription factors: ridiculous or sublime? Nat Cell Biol 2001; 3: E209-E11.

33. Bandyopadhyay D, Mandal M, Adam L, Mendelsohn J, Kumar R. Physical interaction between epidermal growth factor receptor and DNA-dependent protein kinase in mammalian cells. J Biol Chem 1998; 273: 1568-73.

34. Meyn RE, Munshi A, Haymach JV, Milas L, Ang KK. Receptor sig nalling as a regulatory mechanism of DNA repair. Radiother Oncol 2009; 92: 316-22.

35. Krawczyk P, Mlak R, Powrózek T, Nicoś M, Kowalski DM, Wojas-Krawczyk K, Milanowski J. Mechanisms of resistence to reversible inhibitors of EGFR tyrosine kinase in non-small cell lung cancer. Contemp Oncol (Pozn) 2012; 16: 401-6.

36. Chinnaiyan P, Huang S, Vallabhaneni G, Armstrong E, Varambally S, Tomlins SA, Chinnaiyan AM, Harari PM. Mechanism of enhanced radiation response following epidermal growth factor receptor signaling inhibition by erlotinib (Tarceva). Cancer Res 2005; 65: 3328-35.

37. Nyati MK, Morgan MA, Feng FY, Lawrence TS. Integration of EGFR inhibitors with radiochemotherapy. Nat Rev Cancer 2006; 6: 876-85.

38. Rau B, Sturm I, Lage $H$, et al. Dynamic expression profile of p21WAF1/CIP1 and Ki-67 predicts survival in rectal carcinoma treated with preoperative radiochemotherapy. J Clin Oncol 2003; 21: 3391-401.

39. Kopp R, Rothbauer E, Ruge M, et al. Clinical implications of the EGF receptor ligand system for tumour progression and survival in gastrointestinal carcinomas: evidence for new therapeutic options. Recent Results Cancer Res 2003; 162: 115-32. 
40. Giralt J, de las Heras M, Cerezo L, et al. The expression of epidermal growth factor receptor results in a worse prognosis for patients with rectal cancer treated with preoperative radiotherapy. Radiother Oncol 2005; 74: 101-8.

41. Mishani E, Abourbeh G. Cancer molecular imaging: radionuclide-based biomarkers of the epidermal growth factor receptor (EGFR). Curr Top Med Chem 2007; 7: 1755-72.

42. This study was supported by the Scientific Board of Regional Hos pital Liberecand, Research Project UK - LF HK: PRVOUK P37/06.

\section{Address for correspondence}

Igor Richter MD

Department of Oncology, Regional Hospital Liberec

Husova 10

460 63, Liberec, Czech Republic

e-mail: igor.richter@seznam.cz

Submitted: 29.04 .2014

Accepted: 16.07 .2014 